\title{
Distribution Analysis of Rock Fragments Size Based on the Digital Image Processing and the Kuz-Ram Model Cas of Jebel Medjounes Quarry
}

\author{
Mohamed F1,2*, Riadh $\mathbf{B}^{2}$, Abderazzak $\mathrm{S}^{1,2}$, Radouane $\mathrm{N}^{3}$, Mohamed $\mathrm{S}^{2}$ and Ibsa $\mathrm{T}^{2}$ \\ ${ }^{1}$ Laboratory Natural Resources and Planning, Algeria \\ ${ }^{2}$ Faculty of technology, Algeria \\ ${ }^{3}$ Faculty of Earth Science, Algeria \\ *Corresponding author: Mohamed F, Laboratory Natural Resources and Planning, Mining and Geology Department, Algeria
}

Submission: 悳 November 19, 2018; Published: 些 February 28, 2019

\begin{abstract}
Blasting is the most productive excavation method in hard rock mining. In quarries, to remove the mother rock from in-situ and crush into the desired size is realized by bench blasting method. The latter more used in our country because it is a very profitable method. In the quarry of Jebel Medjounes in Ain El-Kebira eastherne Algeria (Setif), the fragmentation of the rocks is done by blasting. The primary purpose of blasting is to fragment rock, and there are significant rewards for delivering a fragmentation size range. Various models have been put forward over the years, attempting to analyse the size distribution resulting from blast designs such as Kuz-Ram model and digital image processing (SplitDesktop® software). The Kuz-Ram model is possibly the most widely used approach to estimating fragmentation from blasting, and the most important function of Kuz-Ram is to guide the blasting engineer in thinking through the effect of various parameters when attempting to improve blasting effects.

In this context, the results of fragmentation such as the rate of oversize fragments were studied using two different methods, the analytical method through the Kuz-Ram model and the numerical method through digital images in an image analysis system called Split Desktop ${ }^{\circledR}$ software. from the results found we note that the value of the oversize of the fragments found by the digital image processing is $11.21 \%$, lower than that found by Kuz-Ram $(31.95 \%)$, which implies that the numerical analysis of the images gives us a better results compared to the Kuz-Ram model, because it is based on the treatment of all the parts of the pile with the different granulometric classes of the rocks.
\end{abstract}

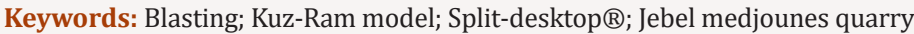

\section{Introduction}

Fragmentation is one of the most important concepts of explosives engineering. Drilling and blasting are the most basis method of rock breaking used in mining operations and civil engineering in Algeria for the purposes such as: breaking of rock for mining; blasting for road construction; digging foundation in hard rocks. In many cases, the blasting work in Algeria, especially blasting in mining engineering, blasting engineers usually deal with the blasting works which quarry out in benches. Blasting is the first step of the size reduction in mining and it is followed by crushing and grinding unit operations. The efficiency of these unit operations is directly related to the size distribution of muckpile. Therefore, reliable evaluation of fragmentation is a critical mining problem.

Rock fragmentation depends on two groups of variables: rock mass properties which cannot be controlled and blasting parameters that can be controlled and optimized. Total cost of aggregate production in a quarry has a minimum value at an optimum fragmentation size [1,2]. Prediction of the optimum fragmentation size will help the quarry owners in selecting blasting parameters to produce required material size at a known cost and in selecting other crushers and conveyor systems. Optimum fragmentation size may not be the required size but knowing the size distribution for blast and rock mass conditions, the contractor can adapt the blasting if possible [2]. For prediction of the fragmentation size after blasting the Kuz-Ram model is generally used. In this study, two analysis methods were conducted at the same rock environment and surface blast design parameters. Kuz-Ram model and Split-Desktop software were utilized as main quantify the size distribution of fragmented rock. The scope of the work is to compare the rate of oversize fragments obtained for the exact same problem from the two methods and to discuss the comparative similarities and differences among them. 


\section{Analysis Method}

Methods to quantify the size distribution of fragmented rock after blasting are grouped as direct and indirect methods. Sieving analysis of fragments is the only technique in direct method. Although it is the most accurate technique among others, it is not practical due to being both expensive and time consuming. For this reason, indirect methods which are observational, empirical and digital methods have been developed.

Observational method which depends on expert's common sense is a widely used technique. An engineer assesses the fragmentation and other blasting results subjectively. This method is not a scientific method as it does not give any information about the size distribution [3-5]. Blasting parameters are considered to determine the size distribution in some empirical models such as Larsson's equation, SveDeFo formula, KUZ-RAM model etc. [5].

The Kuz-Ram model is an empirical fragmentation model based on the [6] Rosin \& Rammler equations modified by $[7,8]$, which derives the coefficient of uniformity in the Rosin \& Rammler equation from blasting parameters. Rock properties, explosive properties, and design variables are combined in this modern version of the Kuz-Ram fragmentation model.

The most popular method to quantify the fragmentation is the determination of the size distribution using digital imaging processing techniques. This method being cheap and useful consumes less time and does not interrupt the production at the site. Due to these reasons, it is preferred widely by explosives engineers. It is the second reliable method after sieve analysis. In this method, image acquired from muck pile, haul truck, leach pile, draw point, waste dump, stockpile, conveyor belt, etc. are delineated automatically by using digital image processing techniques and size distribution of fragmented rocks is determined finally $[9,10]$. There are several software's namely SPLIT, WipFrag, GoldSize, FRAGSCAN, TUCIPS, CIAS, Power Sieve, IPACS, KTH, WIEP, etc. that are commercially available to quantify the size distribution. The accuracy of these systems varies between $2 \%$ to $20 \%[11,12]$.

\section{Description of the split-desktop system}

SPLIT is an image processing program for determining the size distribution of rock fragments at various stages of rock breaking in the mining and processing of mineral resources. The desktop version of SPLIT refers to the user-assisted version of the program that can be run by mine engineers or technicians at on-site locations. The desktop SPLIT system consists of the SPLIT software, computer, keyboard and monitor. There must be a mechanism (software and/ or hardware) for downloading digital or video camera images onto the computer. For digital cameras the software that is supplied with the camera is required and for video camera images a frame grabber board is necessary. For higher resolution images and for ease of image selection, than is available by most frame grabbers, a digital camera is recommended. Resolution of the images should be at least $512 \times 512$. The first step is for the user to acquire images in the field and download these images onto the computer. The source of these images can be a muck pile, haul truck, leach pile, draw point, waste dump, stockpile, conveyor belt, or any other situation where clear images of rock fragments can be obtained. The SPLIT program first assists the user in properly scaling the images. SPLIT can then automatically delineate the fragments in each of the images and determine the size distribution of the rock fragments. SPLIT allows the resulting size distributions to be plotted in various forms (linear-linear, log-linear, log-log, and Rosin Rammler). The size distribution results can also be stored in a tab-delineated file for access in separate spreadsheet and plotting programs [4].

\section{Information about the quarry}

The Limestone quarry was established in 1975 in the Northern of setif in Algeria to provide crushed Limestone aggregate to the cement industry. The quarry runs benches that are approximately $15 \mathrm{~m}$ high. The mine performed percussion drilling that are sized at $110 \mathrm{~mm}$. Blasting was done via the use of an electrical shock tube system. Each hole is loaded with a $79.15 \mathrm{~kg}$ Marmanite and approximately $45 \mathrm{~kg}$ of ANFO per hole for a total of $124.15 \mathrm{~kg}$ of explosives per hole. Blasthole pattern were arranged as staggered three rows. A view of the quarry is given in Figure 1.

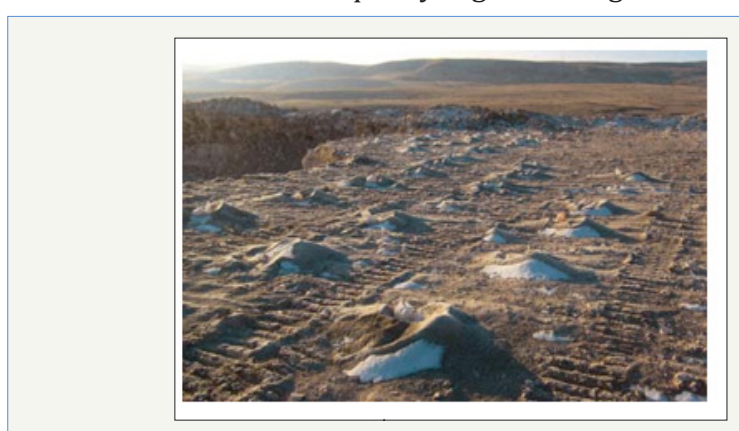

Figure 1: General view of the bench blasthole pattern.

\section{Methodology}

In order to improve the quality of rock fragmentation in the Ain El Kebira quarry, a new blasting plan was proposed. In the proposed blasting plan, the only changing parameter was blasthole inclination in order to improve the fragmentation results. It is intended to investigate the effect of blasthole inclination on fragmentation at the same rock environment and surface blast design parameters. In other words, structural geology of the bench did not vary. Since the limestone is medium to hard rock, MarmaniteIII and ANFO were used as main blasting agents in the existent and proposed blasting plan. First, the rock factor value for the quarry was calculated by the Kuz-Ram model by means of design parameters and fragment size indirectly in the existent and proposed blasting plan. secondly, After the blast, fragmentation was investigated using the Split Desktop® image analysis software in the existent and proposed blasting plan. The investigation procedure is given in Figure 2 as working scheme. Finally, the results of the rate of oversize fragments obtained by two blasting plans (existent and proposed) was compared from the two methods and to discuss the comparative similarities and differences among them. 


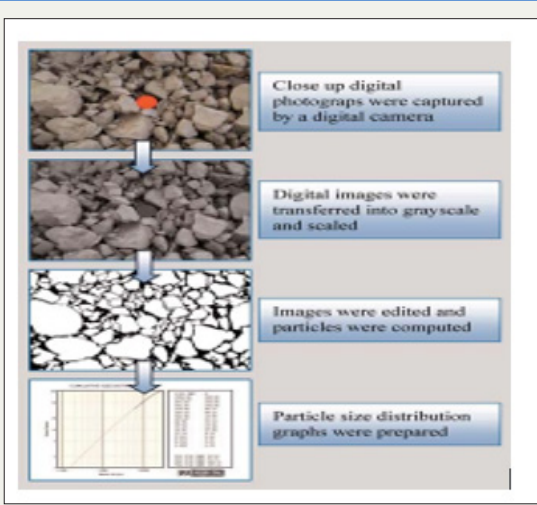

Figure 2: Procedure for the determination of particle size distribution by using Split Desktop ${ }^{\circledR}$ digital image analysis software.

\section{Result and Discussion}

Table 1: The blasting parameters designing for the blasts with the charge diameter of $110 \mathrm{~mm}$.

\begin{tabular}{|c|c|c|}
\hline \multirow{2}{*}{ Parameter } & \multicolumn{2}{|c|}{ Values } \\
\hline & Existant plan & Proposed plan \\
\hline Rock type & \multicolumn{2}{|c|}{ Limestone } \\
\hline Blast hole pattern & \multicolumn{2}{|c|}{ Staggered } \\
\hline Charge Length (m) & 13,45 & 14,2 \\
\hline Blasthole length (m) & 16 & 16,95 \\
\hline Sub dril length $(\mathrm{m})$ & 1 & 1,65 \\
\hline Burden (m) & 4 & 4 \\
\hline Spacing (m) & 4,29 & 4,29 \\
\hline Bench Height (m) & \multicolumn{2}{|c|}{15} \\
\hline Face Dip Direction & $90^{\circ}$ & $80^{\circ}$ \\
\hline Borehole inclination & $90^{\circ}$ & $80^{\circ}$ \\
\hline Powder Factor, kg/t & \multicolumn{2}{|c|}{0,19} \\
\hline Charge Weight per hole (kg/hole) & 117,59 & 124,15 \\
\hline Rock volume broken per blast hole $\left(\mathrm{m}^{3}\right)$ & \multicolumn{2}{|c|}{257,4} \\
\hline
\end{tabular}

In this study, rock factor representing the rock mass properties of a quarry was determined indirectly by the resultant fragmentation of the blasting operation carried out by using the specified blast design parameters. Rock factor was calculated by Kuz-Ram model by means of design parameters and fragment size investigated by SplitDesktop ${ }^{\circledR}$ image analysis software. The blasting parameters for the two blasts are presented in Table 1. The blasts used the delayed-electric detonators; the delay time between rows was $25 \mathrm{~ms}$. After blasting, some blasting results were estimated.

\section{Analyse performed with Kuz-Ram model}

Fragmentation sizes of the broken rock piles were investigated in this way and rate of oversize fragments was calculated by using the Kuz-Ram model and blast ability index (rock factor) of the quarry rock was calculated as 7.14. The simulation of the parameters of the blasting plan proposed with the Kuz-Ram model, confirms the reduction of the oversize rate from $27.2 \%$ (Figure 3 ) for the existing plan to $24.7 \%$ (Figure 4 ) for the proposed plan, which is considered an improvement more or less beneficial for the company if we take into account the amount of limestone extracted per year.

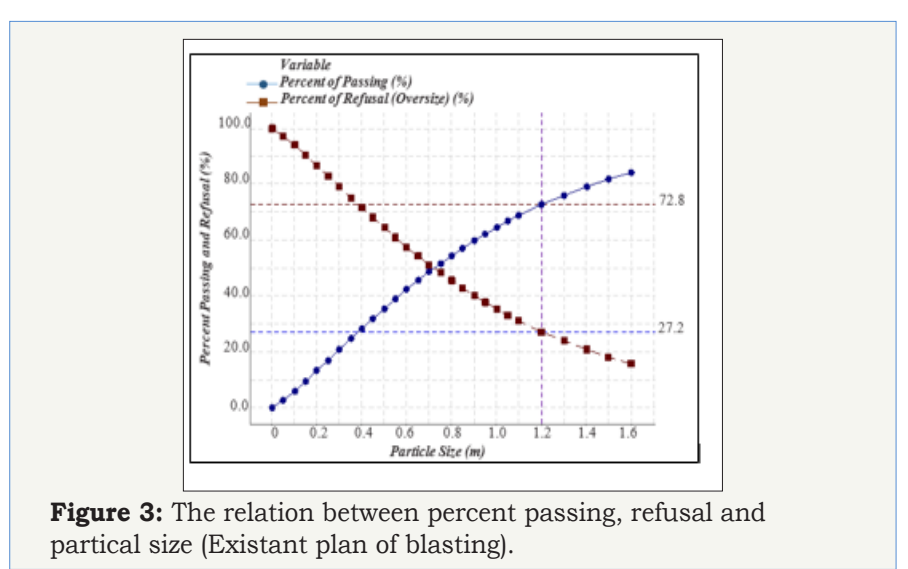

\section{Analyse performed with split desktop ${ }^{\circledR}$ sofware}

The simulation of the parameters of the blasting plan proposed with the Split Desktop ${ }^{\circledR}$ image analysis software, confirms the reduction of the oversize rate from $31.59 \%$ (Figure 5) for the existing plan to $11.21 \%$ for the proposed plan (Figure 6). 


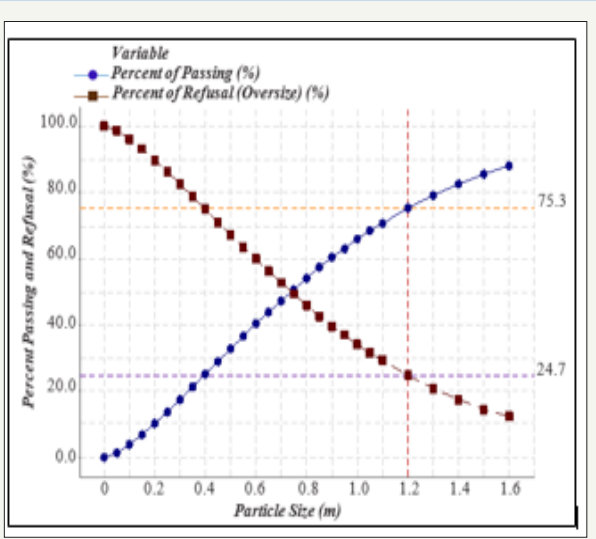

Figure 4: The relation between percent passing, refusal and partical Size (Proposed plan of blasting).

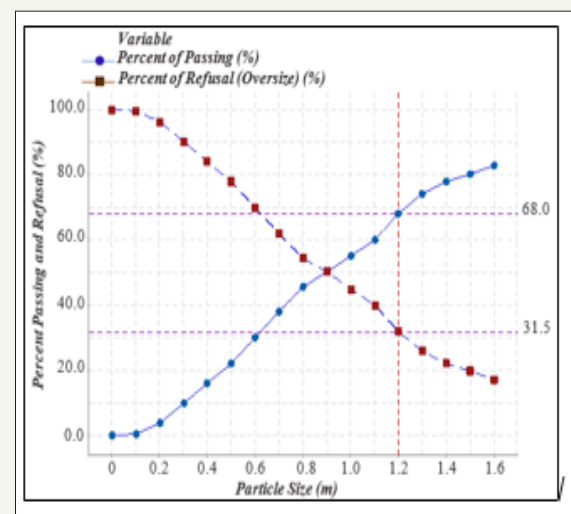

Figure 5: The relation between percent passing, refusal and partical size - existant plan of blasting.

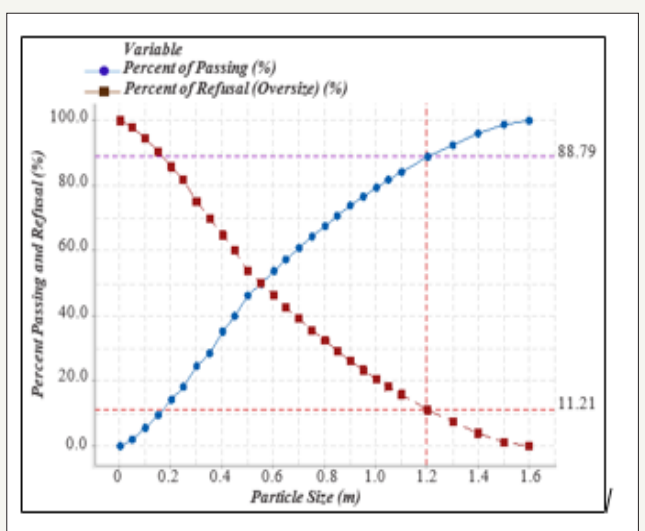

Figure 6: The relation between percent passing, refusal and partical Size-proposed plan of blasting.

\section{Conclusion}

According to the results obtained by the Kuz-Ram model, we note that the rate of oversize of the proposed plan is of a value of $24.7 \%$, lower than the value found for the existing plan $(27.2 \%)$.The digital processing of images is taken of rock fragments in real field (proposed plan) indicates that the rate of oversize is of $11.21 \%$, which is lower than that found for the existing firing plan (31.59\%). The comparison between the results obtained from the analysis of fragmentation quality in the conditions of the quarry of Ain El-Kebira for the existing and proposed plans, with both methods indicates to us the improvement of the fragmentation quality obtained with the proposed plan. Implying that the inclination of the holes plays a very important role in fragmentation quality analysis. If we compare between the two models, we notice that the numerical analysis of the images gives us a better approximation of the results compared to the Kuz-Ram model, because it bases on the treatment of all the parts of the pile with the different granulometric classes rocks (fine, medium and coarse).

\section{References}

1. Mackenzie AS (1967) Optimum blasting. Proc. 28 ${ }^{\text {th }}$ Annual Minnesota Mining Symp. Duluth, USA, pp. 181-188.

2. Morin MA, Ficarazzo F (2006) Monte Carlo simulation as a tool to predict blasting fragmentation based on the Kuz-Ram model. Computers \& Geosciences 32(3): 352-359.

3. Wu X, Kemeny JM (1992) A Segmentation Method for Multi-Connected Particle Delineation, Proc. of the IEEE Workshop on Applications of Computer Vision, IEEE Computer Society Press, Los Alamitos, California, USA, pp. 240-247.

4. Kemeny JM, Devgan A, Hagaman RM, Wu X (1993) Analysis of rock fragmentation using digital image processing. Journal of Geotechnical Engineering 119(7): 1144-1160.

5. Jimeno CL, Jimeno EL, Carcedo FJA (1995) Drilling and Blasting of Rocks, In: Balkema AA, Rotterdam (Eds.), Netherlands, Europe.

6. Kuznetsov VM (1973) The mean diameter of the fragments formed by blasting rock. Soviet Mining Science 9(2): 144-148.

7. Cunningham CVB (1983) The Kuz-Ram model for prediction of fragmentation from blasting. In: Holmberg R, Rustan A (Eds.), Proc. $1^{\text {st }}$ Int Symp on Rock Fragmentation by Blasting, Lulea University Technology, Lulea, Sweden, pp. 439-453.

8. Cunningham CVB (1987) Fragmentation estimations and the KuzRam mode four years on Proc $2^{\text {nd }}$ Int Symp on Rock Fragmentation by Blasting, CT: Society for Experimental Mechanics, Keystone, Colorado, USA, pp. 475-487.

9. Higgins M, BoBo T, Girdner K, Kemeny J Seppala V (1999) Integrated Software Tools and Methodology for Optimization of Blast Fragmentation, Proceedings of the Twenty Fifth Annual Conference on Explosives and Blasting Technique, Nashville, Tennessee, USA, 2: 355-368.

10. Kemeny J, Girdner K, BoBo T (1999) New Advances in Digital Image Analysis Software to Quantify the Size Distribution of Fragmented Rock, minnblast 99: 27-43.

11. Franklin JA, Kemeny JM, Girdner KK (1996) Evolution of Measuring Systems: A review, Proceedings of the Fragblast-5 Workshop on Measurement of Blast Fragmentation, AA Balkema, Montreal, Quebec, Canada, pp. 47-52.

12. Rustan PA (1998) Automatic image processing and analysis of rock fragmentation-comparison of systems and new guidelines for testing the systems, fragblast. International Journal of Blasting and Fragmentation 2(1): 5-23. 
Creative Commons Attribution 4.0 International License

For possible submissions Click Here

Submit Article

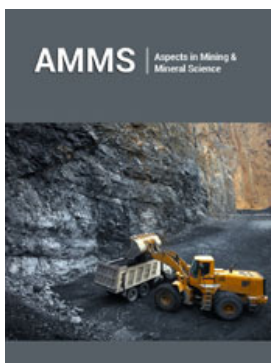

Aspects in Mining \& Mineral Science

Benefits of Publishing with us

- High-level peer review and editorial services

- Freely accessible online immediately upon publication

- Authors retain the copyright to their work

- Licensing it under a Creative Commons license

- Visibility through different online platforms 